\title{
Preliminary Results on Confidence Intervals for Open Bonus Malus
}

\author{
Gracinda R. Guerreiro, João T. Mexia, and Maria F. Miguens
}

\section{Abstract}

Considering open portfolios, we analyze bonus-malus systems (BMS) under a realistic approach, as we already did in Guerreiro and Mexia (Discuss. Math. Probab. Stat. 24(2):197-213, 2004). Using stochastic vortices model we are now able to predict long-run distribution through confidence intervals.

\section{Introduction}

A bonus-malus system (BMS), in automobile insurance, is a rating system from which Insurers, through premiums, are able to, simultaneously, penalize drivers who are responsible for accidents and reward claim-free policyholders. It is, in fact, an a posteriori classification from which the a priori premium is adjusted, according to past experience information. This a posteriori premium aims to better measure the risk that the policyholder represents to the insurer: in the long run, he will pay the premium corresponding to his claim frequency.

The design and evaluation of BMS is based on Markov chains (for detailed presentations of BMS techniques, see [9]). Many authors proposed models for the study of BMS. However, most models are based on the assumption of closed portfolios with a pre-defined entry class for all new policyholders. In Portugal, as stated in $[2,3,6]$, there are many movements among different insurers and frequently, due to commercial goals, a priori discounts are given to new policyholders. This facts clearly reveal unrealistic restrictions in classic models. We consider that analyzing

\footnotetext{
G.R. Guerreiro $(\varangle) \cdot$ J.T. Mexia • M.F. Miguens Departamento de Matemática, Universidade Nova de Lisboa-Faculdade de Ciências e Tecnologia, Campus de Caparica, 2829-516 Caparica, Portugal e-mail: grg@fct.unl.pt; jtm@fct.unl.pt; mfvm@fct.unl.pt
} 
BMS under open portfolio approaches renders a more realistic perspective. For open portfolio models, see, for instance, $[2,6]$.

In this chapter, availing ourselves of the stochastic vortices (SV) model (see [6-8]), we estimate long-run distribution through confidence intervals. In this way, we are able to obtain intervals for bonus scales which can be useful to define optimal and competitive premiums. SV model has already been developed for populations with complex characteristics. In this paper, we focus on BMS application, so the presented model is congruent to it's structure. For general results, see [8].

\section{Stochastic Vortices Model for Bonus Malus Systems}

\subsection{Transition Matrix}

Let us consider:

- A BMS with $s$ bonus classes in one Markov chain communication class

- One recurrent state, representing the withdrawals of policyholders

The one-step transition matrix of the Markov chain will be

$$
\boldsymbol{P}=\left[\begin{array}{cc}
\boldsymbol{K} & \boldsymbol{q}_{1} \\
0 & 1
\end{array}\right]
$$

with

$\boldsymbol{K}-s \times s$ transition matrix between bonus classes

$\boldsymbol{q}_{1}-s$ components vector of annulment probabilities

With $\boldsymbol{q}_{n}=\sum_{j=0}^{n-1} \boldsymbol{K}^{j} \boldsymbol{q}_{1}, n \in \mathbb{N}$, the $n$ step transition matrix will be

$$
\boldsymbol{P}^{n}=\left[\begin{array}{cc}
\boldsymbol{K}^{n} & \boldsymbol{q}_{n} \\
0 & 1
\end{array}\right], \quad n \in \mathbb{N} .
$$

\subsection{Policyholders Entries}

We assume that entries into the portfolio occur at the beginning of time periods, which we will consider as years. Moreover, we assume that:

- Numbers of new policyholders in year $i, E_{i}, i \in \mathbb{N}$, are independent and Poissondistributed random variables with means $\lambda_{i}^{\prime}, i \in \mathbb{N}$.

- Mean values $\lambda_{i}^{\prime}$ are given by

$$
\lambda_{i}^{\prime}=a+b \theta^{i}, a, b \in \mathbb{R}, 0<\theta<1, i \in \mathbb{N} .
$$

Note that we are focusing on $0<\theta<1$, but the model was developed for $\theta>0$; see [7]. Equation (3) represents a quite general assumption and applies to a variety of population entries. We point out the next example: when $a=0$, 
(3) represents a population with a geometric growth on entrances; if $b=-a$ and $\theta=e^{-\delta}, \delta>0$, (3) will represent a population with an asymptotic growth on entrances; for the situations $\theta=1$ or $b=0$, (3) reflects a constant rate on entrances.

- New policyholders are subject to an initial classification. Elements entered in the $i$ th year will be allocated to any of the bonus classes, according to the components of probabilities vector $\boldsymbol{c}_{i}, i \in \mathbb{N}$. We assume that new elements do not leave the portfolio immediately after initial classification; thus $\boldsymbol{c}_{i}^{T}=$ $\left[\boldsymbol{t}_{i}^{T} \mid 0\right]$, with $\boldsymbol{t}_{i}$ corresponding to the probabilities of a new element entering into transient states, in year $i$. For details about initial classification criteria, see [7].

\subsection{Expected Subpopulations Dimension}

Let $N_{i}$ be the number of policyholders initially placed in each bonus class in time period $i, i \in \mathbb{N}$.

The next proposition (see [4]) has a fundamental role in our developments:

Proposition 1. If $E \sim \operatorname{Poisson}(\mu)$ and $(X \mid E=e) \sim \operatorname{Multinomial}(e, c)$ with $\boldsymbol{c}^{T}=$ $\left(c_{1}, \ldots, c_{k}\right)$, then $\boldsymbol{X}$ is a random vector whose margins, $X_{1}, \ldots, X_{k}$, are independent and Poisson-distributed random variables with mean values $\left(\mu_{1}, \ldots, \mu_{k}\right)^{T}=$ $\left(\mu c_{1}, \ldots, \mu c_{k}\right)^{T}$, respectively.

We will say that $\boldsymbol{X}$ has a multivariate Poisson distribution with mean vector $\boldsymbol{\mu}=\left(\mu_{1}, \ldots, \mu_{k}\right)^{T}$ and will be represented by $\boldsymbol{X} \sim \operatorname{Poisson}(\boldsymbol{\mu})$.

Theorem 1. Consider a population with $k$ subpopulations and that the numbers $E_{i}, i \in \mathbb{N}$, of new elements arriving to the population in year $i$ are Poisson distributed with mean value $\lambda_{i}^{\prime}, i \in \mathbb{N}$. New elements are allocated in subpopulations, in year $i, i \in \mathbb{N}$, according to probabilities vector $c_{i}, i \in \mathbb{N}$. After entry, future periodic re-classifications follow stable probability transition matrices. In a time period $m$, the number $\boldsymbol{N}_{i, m}$ of elements in each sub-population, entered in the $i$ th year, will have been subject to $m-i$ reclassifications and are Poisson distributed with parameter

$$
\lambda_{i, m}^{T}=\lambda_{i}^{\prime} \boldsymbol{c}_{i}^{T} \boldsymbol{P}^{m-i}
$$

Proof. According to Proposition 1, we may acknowledge that $\boldsymbol{N}_{i}$, number of elements initially placed in each subpopulation in year $i$, is Poisson distributed with mean vector $\lambda_{i}^{\prime} \boldsymbol{c}_{i}, \boldsymbol{N}_{i} \sim \operatorname{Poisson}\left(\lambda_{i}^{\prime} \boldsymbol{c}_{i}\right)$.

In each time period $m, m \geq i$, the $N_{i, j}, i \in \mathbb{N}, j=1, \ldots, k$, elements entered in year $i$, and initially placed in subpopulation $j$, have been subject to $m-i$ reclassifications and distributed over the subpopulations according to transition 
matrix $\boldsymbol{P}$. The vector of the number of elements $\boldsymbol{N}_{i, m, j}^{T}=\left(N_{i, m, j, 1}, \ldots, N_{i, m, j, k}\right)$, will, according once again to Proposition 1, also be Poisson distributed,

$$
\boldsymbol{N}_{i, m, j} \sim P\left(\lambda_{i}^{\prime} c_{i, j} \boldsymbol{\delta}_{j}^{T} \boldsymbol{P}^{m-i}\right), i, m \in \mathbb{N}, j=1, \ldots, k,
$$

with $\delta_{j}$ a vector whose components are null, except the $j$ th one, which is 1 .

Since the components of $\boldsymbol{N}_{i}, i \in \mathbb{N}$, are independent random variables, vectors $N_{i, m, j}, i, m \in \mathbb{N}, j=1, \ldots, k$, will also be independent. To complete the proof we only need to point out that the vector of total sizes, in time period $m$, will be given by $\boldsymbol{N}_{i, m}=\sum_{j=1}^{k} \boldsymbol{N}_{i, m, j}$. Thus, due to Poisson distribution reproducibility, we obtain $\boldsymbol{N}_{i, m} \sim P\left(\boldsymbol{\lambda}_{i, m}\right)$ with $\lambda_{i, m}^{T}=\sum_{j=1}^{k} \lambda_{i}^{\prime} c_{i, j} \boldsymbol{\delta}_{j}^{T} \boldsymbol{P}^{m-i}=\lambda_{i}^{\prime} \boldsymbol{c}_{i}^{T} \boldsymbol{P}^{m-i}$.

Using (4) (see [7]), we are able to estimate bonus classes dimension, according to stochastic vortices model. The estimator for bonus classes dimension now reflects entrances intensities, initial classification, transition, and annulment probabilities.

For total number of policyholders in each bonus class in time period $m$, we have

$$
\boldsymbol{N}_{m}^{++}=\sum_{i=1}^{m} \boldsymbol{N}_{i, m} \sim P\left(\lambda_{m}^{++}\right)
$$

with

$$
\lambda_{m}^{++T}=\left[\sum_{i=1}^{m} \lambda_{i}^{\prime} \boldsymbol{t}_{i}^{T} \boldsymbol{K}^{m-i} \mid \sum_{i=1}^{m} \lambda_{i}^{\prime} \boldsymbol{t}_{i}^{T} \boldsymbol{q}_{m-i}\right]
$$

\subsection{Asymptotic Results for Transient States}

The existence of stochastic vortices in transient states implies stable limit relative dimension for the bonus classes.

Let us assume that sub-matrix $\boldsymbol{K}$ is a $s \times s$ diagonalizable matrix. Under very general conditions (see [11]), we will have

$$
\boldsymbol{K}=\sum_{j=1}^{s} \eta_{j} \boldsymbol{\alpha}_{j} \boldsymbol{\beta}_{j}^{T} \quad \boldsymbol{K}^{m}=\sum_{j=1}^{s} \eta_{j}^{m} \boldsymbol{\alpha}_{j} \boldsymbol{\beta}_{j}^{T}
$$

with $\eta_{j}\left[\boldsymbol{\alpha}_{j}, \boldsymbol{\beta}_{j}^{T}\right], j=1, \ldots, s$ matrix $\boldsymbol{K}$ eigenvalues [left and right eigenvectors].

Considering the first block of (6), as well as assumption (3), let

$$
\lambda_{m}^{+T}=\sum_{i=1}^{m} \lambda_{i}^{\prime} \boldsymbol{t}_{i}^{T} \boldsymbol{K}^{m-i}=\sum_{i=1}^{m}\left(a+b \theta^{i}\right) \boldsymbol{t}_{i}^{T} \boldsymbol{K}^{m-i}
$$

be the mean vector for transient states (bonus classes), in time period $m$.

Using (7), we identified conditions for convergence of (8). Proposition 2 is established in [7]. For computational simplicity, we obtained last expression in [8]. 
Proposition 2. If entries intensities are given by $\lambda_{i}^{\prime}=a+b \theta^{i}, i \in \mathbb{N},(a, b) \in$ $\mathbb{R}^{2}, \quad 0<\theta<1$ and $\lim _{i \rightarrow+\infty} t_{i, j}=t_{j}, j=1, \ldots, s$, we then have

$$
\lambda_{\infty}^{+T}=\lim _{m \rightarrow+\infty} \lambda_{m}^{+T}=\sum_{j=1}^{s} \frac{\boldsymbol{t}^{T} \boldsymbol{\alpha}_{j} a}{1-\eta_{j}} \boldsymbol{\beta}_{j}^{T}=a \boldsymbol{t}^{T}\left(\boldsymbol{I}_{s}-\boldsymbol{K}\right)^{-1}
$$

This proposition guarantees, under general conditions, the existence of finite limits for the parameters vector of sub-populations in transient states, if $0<\theta<1$.

Long-run distribution of BMS corresponds to limit relative dimensions for transient states, which will be stable as $m \rightarrow+\infty$ (see [7]) and given by

$$
\pi_{\infty, j}=\lim _{m \rightarrow+\infty} \pi_{m, j}=\lim _{m \rightarrow+\infty} \frac{\lambda_{m, j}^{+}}{\sum_{j=1}^{s} \lambda_{m, j}^{+}}=\frac{\lambda_{\infty, j}^{+}}{\sum_{j=1}^{s} \lambda_{\infty, j}^{+}}, j=1, \ldots, s
$$

so a stochastic vortex is established in transient states and long-run distribution for BMS can be easily obtained. Note that initial classification will not interfere in long-run distribution. However, regarding weighted distributions (see [1]), initial classification renders more realistic models.

\subsubsection{Confidence Intervals for Bonus Classes}

Due to (5), for large portfolios we obtain level $q$ confidence intervals for $\lambda_{m, j}^{+}, j=$ $1, \ldots, s, m \in \mathbb{N}$ :

$$
\mathbb{P}\left[N_{m, j}^{+}-z_{q / 2} \sqrt{N_{m, j}^{+}} \leq \lambda_{m, j}^{+} \leq N_{m, j}^{+}-z_{q / 2} \sqrt{N_{m, j}^{+}}\right]=1-\frac{q}{2}
$$

where $z_{q / 2}$ is the upper $1-\frac{q}{2}$ critical value for standard normal distribution.

Using delta method (see [12]), we obtain level $q$ confidence intervals for $\pi_{m, j}, j=1, \ldots, s, m \in \mathbb{N}$ :

$$
\mathbb{P}\left[\frac{N_{m, j}^{+}}{\sum_{j=1}^{k} N_{m, j}^{+}}-z_{q / 2} \sqrt{V_{j} N_{m, j}^{+}} \leq \pi_{m, j} \leq \frac{N_{m, j}^{+}}{\sum_{j=1}^{k} N_{m, j}^{+}}+z_{q / 2} \sqrt{V_{j} N_{m, j}^{+}}\right]=1-\frac{q}{2}
$$

with

$$
V_{j}=\left(\lambda_{\infty}^{+}\right)^{-2}\left[\left(1-\pi_{m, j}\right)^{2}+C^{-2} \pi_{m, j}^{2}-2 C^{-1} \pi_{m, j}\left(1-\pi_{m, j}\right)\right]
$$

or

$$
V_{j}=\left(\lambda_{\infty}^{+}\right)^{-2} \pi_{m, j}^{2}
$$

considering $\lambda_{\infty}^{+}=\sum_{j=1}^{s} \lambda_{\infty, j}^{+}$and $S_{j}=\sum_{i \neq j}^{s} \hat{\lambda}_{m, j}^{+}$.

Equation (12) holds if $\lim _{m \rightarrow+\infty} \sigma_{\lambda_{\infty, j}^{+}} / \sigma_{S_{j}}=C>0$ and (13) holds if, for class $j$, we have $\lim _{m \rightarrow+\infty} \sigma_{\lambda_{j}^{++}} / \sigma_{S_{j}}=0$. 
Table 1 Number of new policyholders per year

\begin{tabular}{llllllllll}
\hline 1997 & 1998 & 1999 & 2000 & 2001 & 2002 & 2003 & 2004 & 2005 & 2006 \\
\hline 4,107 & 9,607 & 15,829 & 22,443 & 29,216 & 34,770 & 39,686 & 32,588 & 46,692 & 49,283
\end{tabular}

\section{$3 \quad$ An Example}

\subsection{Transition Rules and Claim Frequency}

Consider data from a Portuguese insurer portfolio. BMS has $s=20$ classes with premium increasing with class index. For each claim-free year, index decreases by one. The first [each of the next] claim increases the class index by three [five]. The zero bonus/malus class is the tenth.

To $C(t)$, the number of claims in $[0, t)$, we adjusted a mixed Poisson distribution with Gamma structural distribution: $C(t) \sim P(\Lambda), \Lambda \sim \operatorname{Gamma}(\alpha, \beta)$. From the data we obtained the ML estimates $\hat{\alpha}=0.70523$ and $\hat{\beta}=10.10695$.

\subsection{New Policyholders Estimation}

Table 1 resumes insurer information about new policies for automobile insurance.

Let $\left(E_{1}, \ldots, E_{m}\right)$ be the random sample of the number of entries in $m$ consecutive years. Let us assume that $E_{i} \sim P\left(\lambda_{i}^{\prime}\right)$ with $\lambda_{i}^{\prime}=\tau\left(1-e^{-\delta i}\right),(\tau, \delta) \in \mathbb{R}^{2}$. Note that this is a particular case of (3) with $\theta=e^{-\delta}$ and $\tau=-b=a$.

ML estimators for $\tau$ and $\delta$ are the solutions of

$$
\begin{aligned}
& \hat{\tau}=\frac{\sum_{i=1}^{m} e_{i}}{m-\sum_{i=1}^{m} e^{-\hat{\delta} i}} \\
& \hat{\tau} \sum_{i=1}^{m} i e^{-\hat{\delta} i}=\sum_{i=1}^{m} \frac{i e^{-\hat{\delta} i}}{1-e^{-\hat{\delta} i}} e_{i} .
\end{aligned}
$$

From (14) and (15), ML estimates were obtained: $\hat{\tau}=212109$ and $\hat{\delta}=$ 0.026692 . This implies, for general model (3), that $\hat{\theta}=e^{-\hat{\delta}}=0.973661$.

We note that the ML estimate for $\tau$ is unrealistic for this insurer. Due to the Portuguese market and the insurer's quota share, it is not likely that they attain such number of new annual entries. An alternative estimate relies on fix $\tau$ as the insurer's long run perspective on growth and estimate $\delta$, based on that assumption.

\subsection{Initial Classification and Annulment Probabilities}

Initial classification and annulment probabilities were considered not depending on year and estimated from data. Results are presented in Table 2. 
Table 2 Initial classification and annulment probabilities per bonus class

\begin{tabular}{lllllllllll}
\hline$j$ & 1 & 2 & 3 & 4 & 5 & 6 & 7 & 8 & 9 & 10 \\
\hline$c(j)$ & 0.2394 & 0.0537 & 0.1914 & 0.0696 & 0.1886 & 0.0061 & 0.0342 & 0.0104 & 0.0625 & 0.1424 \\
\hline$q(j)$ & 0.1043 & 0.1275 & 0.1542 & 0.1833 & 0.2248 & 0.2179 & 0.2473 & 0.2350 & 0.2375 & 0.4533 \\
\hline$j$ & 11 & 12 & 13 & 14 & 15 & 16 & 17 & 18 & 19 & 20 \\
\hline$c(j)$ & 0.0006 & 0.0004 & 0.0003 & 0.0002 & 0.0002 & $2 \cdot 10^{-5}$ & $3 \cdot 10^{-5}$ & $3 \cdot 10^{-5}$ & $4 \cdot 10^{-6}$ & $2 \cdot 10^{-5}$ \\
\hline$q(j)$ & 0.3909 & 0.4718 & 0.5621 & 0.5964 & 0.5703 & 0.7353 & 0.9487 & 0.4815 & 0.7364 & 0.8276 \\
\hline
\end{tabular}

Table 3 Long run distributions and optimal bonus scales-S. vortices and C. model approaches

\begin{tabular}{lllllll}
\hline$j$ & $\pi_{C}(j)$ & $b_{C}^{G}(j)$ & $\underline{\pi}_{S}(j)$ & $\pi_{S}(j)$ & $\bar{\pi}_{S}(j)$ & $b_{S}^{G}(j)$ \\
\hline 1 & 0.7943 & 0.0531 & 0.6879157898 & 0.6879163605 & 0.6879169313 & 0.06086 \\
\hline 2 & 0.0412 & 0.0764 & 0.0735734532 & 0.0736314416 & 0.0736894300 & 0.07082 \\
\hline 3 & 0.0462 & 0.0996 & 0.0854663157 & 0.0854859575 & 0.0855055993 & 0.08078 \\
\hline 4 & 0.0134 & 0.1228 & 0.0435894748 & 0.0435998164 & 0.0436101579 & 0.09074 \\
\hline 5 & 0.0113 & 0.1461 & 0.0428224948 & 0.0428325975 & 0.0428427002 & 0.10070 \\
\hline 6 & 0.0081 & 0.1693 & 0.0152794230 & 0.0152815824 & 0.0152837419 & 0.11067 \\
\hline 7 & 0.0077 & 0.1926 & 0.0158469193 & 0.0158492070 & 0.0158514948 & 0.12063 \\
\hline 8 & 0.0074 & 0.2158 & 0.0111921268 & 0.0111934917 & 0.0111948565 & 0.13059 \\
\hline 9 & 0.0055 & 0.2390 & 0.0101670336 & 0.0101682137 & 0.0101693938 & 0.14055 \\
\hline 10 & 0.0050 & 0.2623 & 0.0103512971 & 0.0103525116 & 0.0103537261 & 0.15051 \\
\hline 11 & 0.0045 & 0.2855 & 0.0013487107 & 0.0013487707 & 0.0013488308 & 0.16047 \\
\hline 12 & 0.0044 & 0.3088 & 0.0009259372 & 0.0009259720 & 0.0009260068 & 0.17043 \\
\hline 13 & 0.0043 & 0.3320 & 0.0004858670 & 0.0004858807 & 0.0004858945 & 0.18039 \\
\hline 14 & 0.0044 & 0.3552 & 0.0003050116 & 0.0003050186 & 0.0003050255 & 0.19035 \\
\hline 15 & 0.0047 & 0.3785 & 0.0002083131 & 0.0002083171 & 0.0002083212 & 0.20031 \\
\hline 16 & 0.0051 & 0.4017 & 0.0001247669 & 0.0001247688 & 0.0001247707 & 0.21027 \\
\hline 17 & 0.0058 & 0.4250 & 0.0001169801 & 0.0001169818 & 0.0001169835 & 0.22023 \\
\hline 18 & 0.0069 & 0.4482 & 0.0000559784 & 0.0000559790 & 0.0000559796 & 0.23019 \\
\hline 19 & 0.0086 & 0.4714 & 0.0000377499 & 0.0000377502 & 0.0000377506 & 0.24016 \\
\hline 20 & 0.0113 & 0.4947 & 0.0000793801 & 0.0000793812 & 0.0000793823 & 0.25012 \\
\hline & & & & & &
\end{tabular}

Note that policyholders entered through all classes and a large number of insured nullified his policy when arrived to maluses classes. This highlights that assuming closed models and a "starting class" for all new policyholders in rather unrealistic.

\subsection{Long Run Distribution and Optimal Bonus Scale-Stochastic Vortices and Closed Model Approach}

Using (10) for SV model and classical results for BMS (see [9]), long-run distributions were obtained. For SV model we are able to predict long-run distribution through confidence intervals using (11). Following [5] after [10], an optimal bonus scale was obtained for each approach. 
Table 3 presents results for both models. Indexes $C$ and $S$ refer to closed and SV model, respectively. $b^{G}$ represents Gilde and Sundt's linear optimal bonus scale and $\underline{\pi}_{S}(j)$ and $\bar{\pi}_{S}(j)$ the $95 \%$ confidence intervals. Note that long-run distributions differ significantly and closed model overestimates probabilities in maluses classes as well as in higher discount class. This, naturally, has impacts on optimal bonus scales.

With the ML estimates for $\tau$ and $\delta$, the SV model converges slowly to stationarity. This implies that Borgan et al. [1], optimal bonus scale should be implemented instead of Norberg's [10]. In this chapter we illustrate Norberg's optimal bonus scale in order to evaluate portfolio performance in a long-run perspective.

Acknowledgments This work was partially supported by Financiamento Base 2009 ISFL-1-297 from FCT/MCTES/PT.

\section{References}

1. Borgan, Ø., Hoem, J., Norberg, R.: A nonasymptotic criterion for the evaluation of automobile bonus system. Scand. Actuar. J. 3, 165-178 (1981)

2. Centeno, L., Andrade e Silva, J.: Bonus systems in open portfolio. Insurance Math. Econom. 28, 341-350 (2001)

3. Denuit, M., Dhaene, J.: Bonus-malus scales using exponential loss functions. Blatter der DGVFM 25(1), 13-27 (2001)

4. Feller, W.: An Introduction to Probability Theory and it's Applications, 2nd edn. Wiley, New York (1966)

5. Gilde, V., Sundt, B.: On bonus systems with credibility scales. Scand. Act. J. 2, 13-22 (1989)

6. Guerreiro, G.R., Mexia, J.T.: An alternative approach to bonus malus. Discuss. Math. Probab. Stat. 24(2), 197-213 (2004)

7. Guerreiro, G.R., Mexia, J.T.: Stochastic vortices in periodically reclassified populations. Discuss. Math. Probab. Stat. 28(2), 209-227 (2008)

8. Guerreiro, G.R., Mexia, J.T, Miguens, M.F.: A model for open populations subject to periodic re-classifications. J. Stat. Theory Pract. 4(2), 303-321 (2010)

9. Lemaire, J.: Bonus-Malus Systems in Automobile Insurance. Kluwer, Boston (1995)

10. Norberg, R.: A credibility theory for automobile bonus system. Scand. Act. J. 2, 92-107 (1976)

11. Schott, J.R.: Matrix Analysis for Statistics, Wiley Series in Probability and Statistics. Wiley, New York (1997)

12. Tiago de Oliveira, J.: The delta-method for obtention of asymptotic distributions-Applications. Publ. de l'Inst. de Stat. de l'Univ. de Paris 27, 49-70 (1982) 